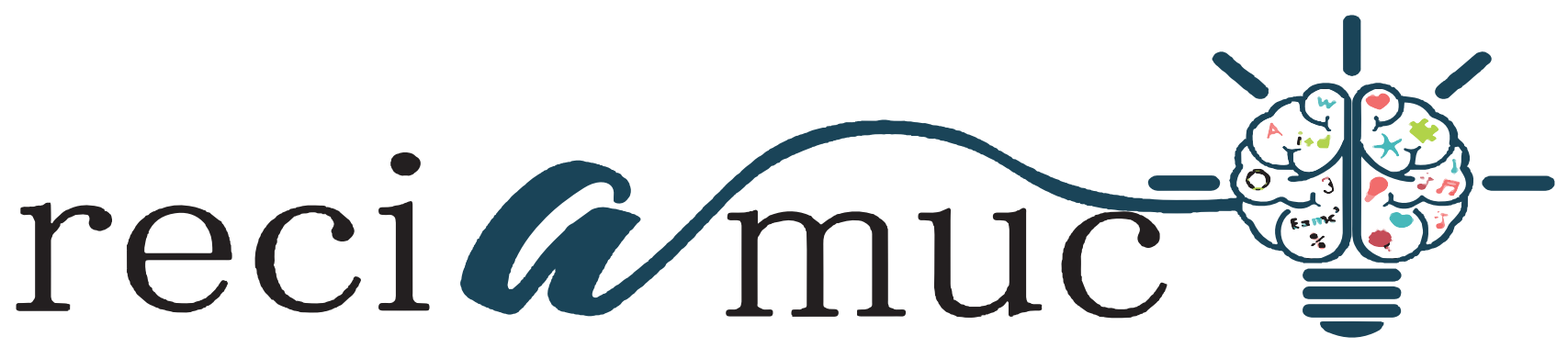

DOI: 10.26820/reciamuc/5.(4).noviembre.2021.2-12

URL: https://reciamuc.com/index.php/RECIAMUC/article/view/726

EDITORIAL: Saberes del Conocimiento

REVISTA: RECIAMUC

ISSN: 2588-0748

TIPO DE INVESTIGACIÓN: Artículo de Revisión

CÓdIGO UNESCO: 32 Ciencias Médicas

PAGINAS: 2-12

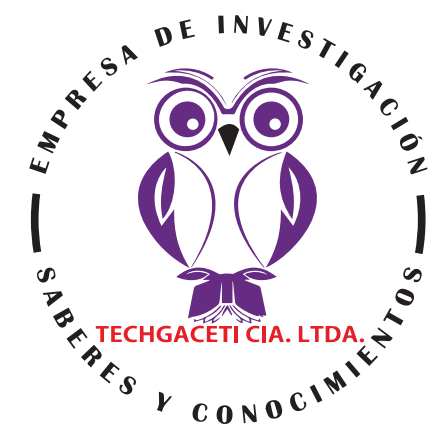

\title{
Entornos Virtuales como soporte en la educación on-line durante la pandemia
}

\author{
Virtual Environments as support in online education \\ during the pandemic
}

Ambientes virtuais como suporte na educação online durante a pandemia

\section{Littelton Roberto Yance Tutiven'; María Elena Ruiz Salazar²; Verónica del Rocío Salazar Palacios ${ }^{3}$}

RECIBIDO: 15/09/2021 ACEPTADO: 05/10/2021 PUBLICADO: 29/11/2021

1. Ingeniero en Sistemas de Calidad y Emprendimiento; Investigador Independiente; Guayaquil, Ecuador; Iyancet@gmail.com; iD https://orcid.org/0000-0001-8554-7838

2. Magister en Diseño y Gestión de Marcas; Ingeniera en Diseño Gráfico; Diseñador Gráfico; Instituto Tecnológico Superior Guayaquil, Ecuador; mruiz@itsgg.edu.ec; iD https://orcid.org/0000-0003-4502-6063

3. Magister en Planificación Evaluación y Acreditación de la Educación Superior; Licenciada en Ciencias de la Educación Especialización: Químico Biológicas; Profesor de Segunda Enseñanza Especialización Químico Biológicas; Administradora Educativa; Ministerio de Educación; Ecuador; delrocio.salazar@educacion.gob.ec;

(i) https://orcid.org/0000-0002-5268-0568

\section{CORRESPONDENCIA}

Littelton Roberto Yance Tutiven lyancet@gmail.com

Guayaquil, Ecuador

(c) RECIAMUC; Editorial Saberes del Conocimiento, 2021 


\section{RESUMEN}

El proceso de formación educativa ha sido constantemente sometido a evaluaciones y reformas. La discusión sobre la transformación educativa, la modernización de las instituciones y la innovación de las aulas ha llevado a una revolución educativa que lleva al menos 25 años desarrollándose y adaptándose en aquello que hoy conocemos como la educación del millenium o educación del Siglo XXI. El desarrollo de los Entornos virtuales ha sido una práctica implementada para expandir el conocimiento y forma una parte importante del crecimiento de la educación on-line. Dada la necesidad de migrar la practica educativa a un entorno seguro, a raíz del brote pandémico del virus SARS-Cov-2, con características netamente virtuales hemos de hacer una investigación bibliográfica que permita el análisis de contenido documental generado no solo durante el año 2019 a raíz de la aparición del SARS-Cov-2, sino una breve orientación de las experiencias sobre el uso y desarrollo de las TIC dentro de los procesos educativos desde los inicios del milenio. El desarrollo de la educación on-line no solo depende de la preparación docente en generar contenido y estrategias que permitirán la transferencia de conocimientos e incentive al estudiante a ser partícipe de la adquisición de los mismos, también depende de una plataforma educativa pública, privada o mixta que pueda garantizar condiciones igualitarias para la comunidad académica que desarrolla precisamente sus entornos virtuales.

Palabras clave: Educación on-line, entornos virtuales, internet, educación del siglo XXI, TIC.

\section{ABSTRACT}

The educational training process has been constantly subjected to evaluations and reforms. The discussion about the educational transformation, the modernization of institutions and the innovation of the classrooms has led to an educational revolution that has been developing and adapting for at least 25 years in what we now know as millennium education or education of the 21st century. The development of virtual environments has been a practice implemented to expand knowledge and is an important part of the growth of online education. Given the need to migrate educational practice to a safe environment as a result of the pandemic outbreak of the SARS-Cov-2 virus with purely virtual characteristics, we have to carry out a bibliographic research that allows the analysis of documentary content generated not only during the year 2019 as a result of the appearance of SARS-Cov-2 but a brief orientation of the experiences on the use and development of ICT within educational processes since the beginning of the millennium. The development of online education not only depends on the teacher preparation to generate content and strategies that allow the transfer of knowledge and encourage the student to be a participant in their acquisition, it also depends on a public, private or educational platform. mixed that can guarantee equal conditions for the academic community that develops precisely its virtual environments.

Keywords: Online education, virtual environments, internet, 21st century education, ICT.

\section{RESUMO}

O processo de formação educacional tem sido constantemente submetido a avaliações e reformas. A discussão sobre a transformação educacional, a modernização das instituições e a inovação das salas de aula tem levado a uma revolução educacional que vem se desenvolvendo e se adaptando há pelo menos 25 anos no que hoje conhecemos como educação do milênio ou educação do século XXI. O desenvolvimento de ambientes virtuais tem sido uma prática implementada para expandir o conhecimento e é uma parte importante do crescimento da educação online. Dada a necessidade de migrar a prática educacional para um ambiente seguro em decorrência do surto pandêmico do vírus SARS-Cov-2 com características puramente virtuais, temos que realizar uma pesquisa bibliográfica que permita a análise do conteúdo documental gerado não só durante o ano de 2019 como resultado do aparecimento do SARS-Cov-2 mas uma breve orientação das experiências de uso e desenvolvimento das TIC nos processos educativos desde o início do milênio. O desenvolvimento da educação online não depende apenas da preparação do professor para gerar conteúdos e estratégias que permitam a transferência de conhecimentos e incentivem o aluno a ser participante na sua aquisição, depende também de uma plataforma pública, privada ou educacional. mista que pode garantir condições de igualdade para a comunidade acadêmica que desenvolve justamente seus ambientes virtuais.

Palavras-chave: Educação online, ambientes virtuais, internet, educação do século 21, TIC. 


\section{Introducción}

El proceso de formación educativa ha sido constantemente sometido a evaluaciones y reformas. La discusión sobre la transformación educativa, la modernización de las instituciones y la innovación de las aulas ha llevado a una revolución educativa que lleva al menos 25 años desarrollándose y adaptándose en aquello que hoy conocemos como la educación del millenium o educación del Siglo XXI.

En primera instancia la incorporación de las Tecnologías de Información y comunicación (TIC) en la Educación Superior además del desarrollo de un espacio Europeo de Educación Superior (EEES) ha incentivado a las universidades enrumbarse en nuevos caminos y direcciones amparados, por supuesto, en la discusión sobre la adquisición de conocimiento cuyas propuestas abogan por que el estudiante sea centro y protagonista del proceso de aprendizaje, y se sustituya la importancia de la enseñanza y la adquisición de conocimiento por la importancia del aprendizaje y la adquisición de competencias. Se trata por tanto no solo de enseñar, sino también de hacer que los estudiantes aprendan. Saber como aprender, cómo dedican su tiempo y su esfuerzo a aprender, y facilitar su aprendizaje, se convierte en prioridad de la universidad del siglo XXI. (Bautista Pérez, Borges Sáiz, \& Forés i Miravalles, 2006)

La virtualización de los ambientes educativos implica la representación de procesos y objetos, que permite al usuario realizar diversas operaciones a través de Internet, y garantizan los procesos de enseñanza y aprendizaje interactivo, inmediato y personalizado en un ambiente caracterizado por la flexibilidad en espacio y tiempo. (Najar S., Alvarado G., \& Otalora L, 2009)

Para las generaciones siguientes ha sido diferente y ha resultado complejo aplicar la formación virtual debido al cambio del contexto pasando de la formación presencial a la virtual o semivirtual. La docencia se ha preocupado por formarse y atreverse a realizar investigaciones y reflexiones sobre la propia formación, sobre las maneras de enseñar y facilitar el aprendizaje. Investigar en formación significa verse, reconocerse, reflexionar y comprender el rol docente a través de la mirada de los propios estudiantes. (Bautista Pérez, Borges Sáiz, \& Forés i Miravalles, 2006)

El e-learning no trata solamente de tomar un curso y colocarlo en un ordenador, se trata de una combinación de recursos, interactividad, apoyo y actividades de aprendizaje estructuradas. La formación virtual utiliza un software específico denominadas genéricamente plataformas de formación virtual. Existen diferentes grupos de entornos de formación según la finalidad de los mismos. El tipo de entorno o sistema adecuado para el e-learning, son los sistemas de gestión del conocimiento (LMS) o Entornos Virtuales de Aprendizaje (EVA), estos son una agrupación de las partes más importantes de los demás entornos para aplicarlos en el aprendizaje. Los EVA se podrían describir como entornos que:

- Permiten el acceso a través de navegadores, protegido generalmente por contraseña o cable de acceso.

- Utilizan servicios de la web 1.0 y 2.0.

- Disponen de un interface gráfico e intuitivo. Integran de forma coordinada y estructurada los diferentes módulos.

- Presentan módulos para la gestión y administración académica, organización de cursos, calendario, materiales digitales, gestión de actividades, seguimiento del estudiante, evaluación del aprendizaje.

- Se adaptan a las características y necesidades del usuario. Para ello, disponen de diferentes roles en relación a la actividad que realizan en el EVA: administrador, profesor, tutor y estudiante. Los privilegios de acceso están personalizados y dependen del rol del usuario. De modo que, el EVA debe de adaptarse a las necesidades del usuario particular.

- Posibilitan la comunicación e interacción entre los estudiantes y el profesortutor.

- Presenta diferentes tipos de actividades que pueden ser implementadas en un curso.

- Incorporan recursos para el seguimiento y evaluación de los estudiantes. (Belloch, 2012)

La pandemia por coronavirus (COVID-19) que ha generado una crisis sanitaria está repercutiendo en diferentes ámbitos de la sociedad; la educación no queda exenta de las consecuencias de este problema sanitario al verse afectados 1,600 millones de alumnos en más de 190 países en todos los continentes según datos de la ONU en 2020. En el mundo se han suspendido clases en todos los niveles educativos, por lo que las escuelas, los docentes, los estudiantes y el personal administrativo están enfrentando el reto de continuar el proceso formativo desde 
el hogar de cada estudiante, con apoyo de los recursos digitales lo que nos hace mirar hacia la educación en situaciones de crisis. (Delgado Sánchez \& Martínez Flores, 2021)

La presente investigación pretende recopilar los análisis y conclusiones relevantes de estudios e investigaciones acerca de las experiencias ganadas para los Entornos Virtuales en medio de la contingencia sanitaria a razón de la pandemia por Covid-19 en el proceso educativo on-line.

\section{Metodología}

Desde un enfoque interpretativo la realización de revisión bibliográfica presente se sustentó en el paradigma cualitativo, dada su orientación hacia el análisis de contenido documental generado no solo durante el año 2019 a raíz de la aparición del SARS-Cov-2 sino una breve orientación de las experiencias sobre el uso y desarrollo de las TIC dentro de los procesos educativos desde los inicios del milenio.

La búsqueda de información se limita al idioma español, en donde se identifica, caracteriza, clasifica, ordena, y seleccionan los libros, articulos, publicaciones de actas de conferencias entre las que se consideren relevantes para el tema de estudio siempre que se pueda verificar la validez y soporte científico-académico.

Para la recolección de las fuentes bibliográficas, se aplicó el modelo búsqueda avanzada a través de las palabras clave, todas relacionadas con los términos: entornos virtuales de aprendizaje, educación durante la pandemia, educación on-line. Así mismo, se reunió un número importante de publicaciones que se ajustaron a las variables en estudio, a los objetivos de la investigación y los criterios establecidos por lo que se procedió al análisis, resumen y selección de los datos destacados para la consecución de los objetivos planteados en la investigación.

\section{Resultados}

La función académica universitaria siempre se ha entendido como un binomio indisoluble de docencia e investigación. Desde el inicio de la capacitación pedagógica venía dada o por la formación requerida, por las ganas de aprender a enseñar, o por el aprendizaje vicario. Muchos profesores universitarios no han tenido la oportunidad de realizar un aprendizaje vicario, ni se han formado en Entornos Virtuales de Enseñanza y Aprendizaje (EVEA) dado que no existía el internet o porque no formaron parte de sus tareas académicas. Tanto los docentes como la propia universidad son claves en ayudar a que sus estudiantes tengan una cierta preparación como estudiantes en línea, de una forma transversal, más allá de los contenidos, destrezas y actitudes propias de los estudios que cursen. (Bautista Pérez, Borges Sáiz, \& Forés i Miravalles, 2006)

Hay que tener en cuenta que en la sociedad del conocimiento el rol del docente se vuelva cada vez más importante, al igual que el desarrollo del material didáctico, la evaluación como componentes de un proceso educativo responden cada vez a la incorporación de las $\mathrm{TIC}$, esto implica que las transformaciones estructurales sean necesarias para que se pueda dar respuesta a las exigencias de un aprendizaje permanente y al requerimiento de las nuevas competencias. (Najar S., Alvarado G., \& Otalora L, 2009)

En el CSCL (Computer Supported Collaborative Learning) consideran que los tres tipos de interacción: cognitiva, social y organizativa favorecen el aprendizaje individual, tanto el relativo a los aprendizajes propios de la materia como el desarrollo de habilidades para colaborar en el futuro. Los estudiantes perciben que la activación de la interacción en los tres niveles se alinea con la motivación y favorece que se sientan más integrados en el estudio de la asignatura. La concepción del aprendizaje como un proceso de interacción y relación está establecida en una vasta tradición ligada a corrientes pedagógicas como el constructivismo, socioconstructivismo o el aprendizaje social. Sin embargo, en el ámbito universitario es habitual que se asocie el aprendizaje en exclusiva al nivel cognitivo, eludiendo la interacción social que media estos procesos y que resulta fundamental en nuestras Ecologías de Aprendizaje, articuladas en torno a la interacción humana y tecnológica. La interrelación natural de los tres tipos de interacción va a ser la red en la que se sostendrán nuestros estudiantes en el futuro profesional, donde la cognición será un elemento importante. Pero, al igual que sucede en el trabajo grupal en el ámbito educativo, será necesario que hayan adquirido competencias para cooperar y educar. (Hernández-Sellés, 2021)

Las aulas de los campus virtuales establecen Área y Adell (2009), son el sitio dónde se enseña y se aprende, un espacio o entorno creado virtualmente con la intencionalidad de que un estudiante obtenga experiencias de aprendizaje a través de recursos/materiales formativos bajo la supervisión e interacción con un profesor. Son las personas, no las tecnologías, quie- 
nes deciden sobre los sentidos atribuidos a estos espacios de formación y sobre las formas de circulación de los saberes. Las aulas virtuales como soportes tecnológicos al teletrabajo docente son espacios dónde es posible enseñar de manera ubicua, omnipresente. No son nuevos espacios ni son los únicos donde se enseña y aprende en la virtualidad, pero son institucionales. En la educación superior, la formación en ambientes virtuales o entornos tecnológicos es anterior data y es reconocida como aprendizaje electrónico. Los medios de comunicación la nombran como educación a distancia, cuyos inicios son previos al surgimiento de los medios masivos y de las TIC. (De Luca, 2020)

Sin embargo, cada formador/a de docentes afronta la urgencia de enseñar en aulas virtuales con disposiciones y un bagaje de experiencias previas tan diversas como las estudiantiles. Un problema sería que la necesidad de enseñar en espacios virtuales sin saber cómo usar la plataforma institucional y sus aplicaciones, más el empeño en saber hacer, redujeran la propuesta a la mirada tecno-centrista. Mientras que lo importante sería no centrarse en la tecnología, sino integrarla estratégicamente, partiendo de reconocer el imaginario, las habilidades y las barreras tecnológicas presentes en formadores y docentes en formación, para planificar la formación articulando saberes disciplinares, estrategias y nuevas prácticas culturales con las potencialidades de las aulas virtuales. (De Luca, 2020)

La UNESCO (2020) anticipaba el aumento de los conflictos armados, los desastres naturales y las epidemias en distintas partes del planeta, todos ellos vulnerando y en algunos casos impidiendo la continuidad educativa de millones de niños, adolescentes y adultos. En este contexto, cobra especial importancia la sugerencia de Quirós (2009): promover a gran escala el uso de los recursos didácticos digitales por profesores y estudiantes, para favorecer el aprendizaje colaborativo en dicho entorno. Los entornos virtuales de aprendizaje (EVA), entre los que se encuentra el uso de plataformas e-learning, comprenden una amplia variedad de recursos, como la plataforma Moodle, Flipped Classroom, Google Classroom, Edmodo, Blackboard-collaborate, así como diversos recursos disponibles en la Web, que fueron concebidos solo con un fin comunicacional. (Delgado Sánchez \& Martínez Flores, 2021)

Si bien el uso de tecnología aplicada a la educación ha brindado la posibilidad de crear formas de acceso al conocimiento distintas a las convencionales, en el contexto latinoamericano aún se vivía un periodo de transición, pues aunque conceptos como Entornos virtuales de aprendizaje (EVA), E-learning, M-learning, B-learning, tecnologías de la información y comunicación (TIC) entre otros, cobraban cada vez más fuerza en el ámbito educativo, la mayoría de países, en Latinoamérica no estaban listos para afrontar un cambio total, en su modelo pedagógico, sin embargo ante la gravedad de la crisis sanitaria se vieron obligados a transitar hacia un modelo pedagógico de enseñanza virtual basado en la aplicación de las (TIC). (Najar S., Alvarado G., \& Otalora L, 2009)

En el contexto descrito cada sistema educativo debe tener claro que para que el modelo pedagógico asumido tenga éxito deben centrar sus acciones en superar tres aspectos fundamentales: La Implementación de recursos tecnológicos, capacitación del personal docente, adaptación de los estudiantes a esta forma de educación, sin olvidar que cada uno de estos aspectos deben enfocarse en buscar la calidad educativa. En Latinoamérica según datos del Internet world stats representa el 67\% del total de la población. (Najar S., Alvarado G., \& Otalora L, 2009)

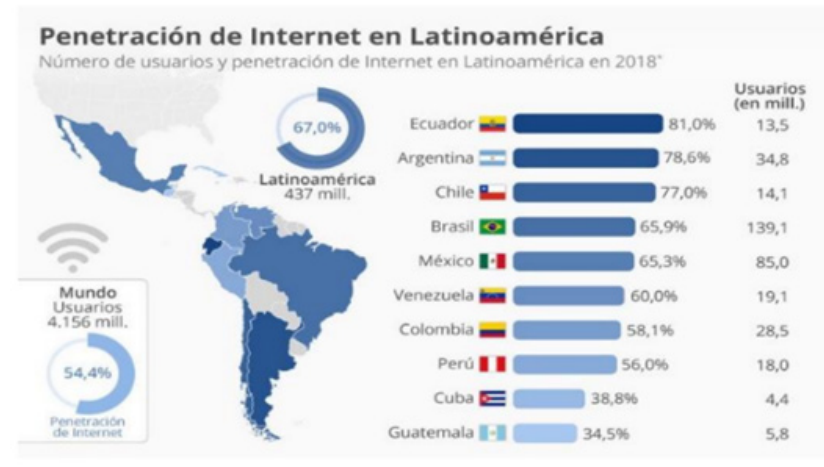

Gráfico 1. Penetración de Internet en Latinoamérica2018

Fuente: Internet world stats

La incorporación de la informática y las telecomunicaciones han permitido que en el conocimiento se haya generado nuevos paradigmas en la incursión de los entornos virtuales educativos como herramientas tecnológicas que han permitido generar otros escenarios sin fronteras para poder dar a conocer conocimiento y de igual manera poderlo gestionar mejor. La implantación de las Tecnologías de la Información y la Comunicación han permitido que la implantación de la Gestión de Conocimiento (GC) no 
acabe pues con su incorporación ha generado otro valor en la sociedad permitiendo la realización de diversas actividades de aprendizaje en forma interactiva, creativas potenciando las habilidades de los estudiantes para la solución de problemas. La implantación de la GC desde esta propuesta permite indicar cuáles son algunas de las condiciones bajo las cuales se intenta dar respuesta al impulso de una sensibilización cultural que permite trabajar en un ambiente colaborativo y cooperativo, con facilidad a la generación, acceso y aplicabilidad del conocimiento desde lo que se sabe hacer, se hace y se comparte. Esto es lo que permite optimizar el ciclo de experiencia- aprendizaje. (Najar S., Alvarado G., \& Otalora L, 2009)

Si bien el uso de tecnología, aplicada en el campo educativo ha propuesto la contingencia de crear formas de acceso al conocimiento, diferentes a las cotidianas, en el contexto latinoamericano aún se vivía una etapa de transformación, a pesar de que concepciones como Entornos virtuales de aprendizaje (EVA), E-learning, M-learning, B-learning, tecnologías de la información y comunicación (TIC) entre otros, cobraban cada vez más fuerza en el ámbito educativo, la mayoría de países en Latinoamérica no estaban listos para afrontar un cambio total en su modelo pedagógico (Borba, 2018). Sin embargo ante la gravedad de la crisis sanitaria se vieron obligados a transitar hacia un modelo pedagógico de enseñanza virtual basado en la aplicación de las (TIC) (Hardman, 2019). En referencia a los Entornos Virtuales de Aprendizaje, se ha podido determinar que las tecnologías e-learning ayudan a compartir el conocimiento, y que, si bien esto es cierto en términos generales, lo es también de forma particular en organizaciones y empresas que desean aumentar sus capacidades. Así, el uso del e-learning para desarrollar canales para compartir el conocimiento dentro de la organización ayuda a conseguir la excelencia y la competitividad aumentando las capacidades de tipo dinámico y el potencial innovador (Iris y Vikas, 2011). (Sornoza Pico \& Yánez Rodriguez, 2020)

Las tecnologías e-learning agrandan la capacidad para enfrentar los cambios rápidos e intensivos del conocimiento, brindando la oportunidad de favorecer de forma eficaz en compartir el conocimiento. Vale hacer mención entonces que, la formación virtual utiliza un software específico denominadas genéricamente plata- formas de formación virtual. Existen diferentes grupos de entornos de formación según la finalidad de los mismos. Sin embargo, el que más se utiliza son los EVA (Baelo, 2009). (Sornoza Pico \& Yánez Rodriguez, 2020)

Para los docentes la experiencia virtual no era parte de sus prácticas educativas habituales. La virtualidad sumó complicaciones no planificadas, aunque también abrió nuevas posibilidades para el abordaje de la práctica pedagógica. Para la otra parte la experiencia educativa en entornos virtuales ya era una práctica sostenida, pero lo atípico de la situación se localizó en la aparición de problemáticas antes no contempladas. Entre posibilidades y problemas, oportunidades y proyectos, se construyeron nuevas experiencias relacionadas con la formación docente para el trabajo en los entornos virtuales, el acompañamiento institucional en la experiencia educativa en la virtualidad, las condiciones de acceso a tecnologías, las herramientas y conocimientos para el desarrollo de clases en entornos virtuales, las condiciones de trabajo en aislamiento y las experiencias de intercambios con estudiantes en el desarrollo de actividades de educación virtual. (Ardini, Montserrat Herrera, González Angeletti, \& Secco, 2020)

La enseñanza en tiempos de pandemia es una práctica social compleja que no responde a una única receta y que forma una trama con la presente situación que nos desafía a enseñar en aulas virtuales. En la era digital, la clásica concepción de educación a distancia debía ser matizada y redefinida porque los espacios y aulas virtuales ya no se usaban exclusivamente en la formación a distancia, sino que también se empleaban en la educación presencial En el campo educativo, el teletrabajo o trabajo mediado por tecnologías, se conoce como e-learning o aprendizaje electrónico. Este se refiere a algún tipo de proceso de enseñanza y de aprendizaje realizado con dispositivos electrónicos conectados a internet (Área y Adell, 2009). (De Luca, 2020)

La literatura académica reconoce modos intermedios llamados aprendizaje flexible, híbrido, blended learning o semi-presencial, y modos presenciales con distintos grados de interacción, cuyas aulas virtuales se configuran como recurso complementario, ampliado y expandido de la clase presencial. Además, se reconocen dos categorías de usos de las TIC en la ense- 
ñanza: como entorno o ambiente, y como medio o herramienta. En la formación presencial, cuando las TIC se usan como medios para la enseñanza, quien enseña es quien en distintos momentos de la clase presenta los recursos en soportes digitales (un PowerPoint, un video o una imagen digital, etc.) como antes se presentaban en soportes analógicos (retroproyector, películas, fotos). Esto no quita que los recursos provistos por estos medios puedan almacenarse en un entorno personal en la nube (un blog, un drive) con acceso por internet. Por el contrario, cuando configuramos un aula virtual como entorno, o como sitio principal de formación, quien la configura y quienes acceden a sus recursos y experiencias didácticas para aprender sobre la disciplina en cuestión, pueden hacerlo en cualquier momento y desde cualquier lugar, sin la intermediación ni la presencia del formador que guía el aprendizaje con intervención indirecta y con tutoría. (De Luca, 2020)

En el uso de las TIC como entorno, también están presentes la herramienta de las apps (aplicaciones) y medios o recursos didácticos (una infografía, un mapa conceptual o mental, una línea de tiempo, etc.). Cualquiera que sea la modalidad de aprendizaje electrónico, en las universidades se utiliza la plataforma virtual moodle, mientras que los institutos superiores tienen su nodo en la plataforma educativa, y en los niveles obligatorios el sistema educativo ofrece google classroom, una plataforma abierta y "amigable" de uso intuitivo. No podemos obviar que la teleformación es una nueva función del profesorado con muchas más horas de trabajo, a veces invisible para el planeamiento de los sistemas educativos, además que esta sobrecarga amplía la brecha digital perjudicando a quienes tienen la doble carga de tareas laborales y familiares. (De Luca, 2020)

Enfocar la didáctica incluye cuidar del otro cuando se configuran las situaciones de enseñanza y de aprendizaje de la propuesta pedagógica, atendiendo a la brecha digital que afecta a muchos, pero que no impide enseñar con tecnologías. También implica tomar decisiones que consideren a las familias sin computadora, o con una compartida y sin conectividad. Lo que produce la tecnología en la educación es ampliar las posibilidades de elección de un profesor que tiene que tomar decisiones sobre cómo enseñar (Bates, 2009: 109). (De Luca, 2020)
Si bien la autopercepción del nivel de formación es más alto que bajo el objetivo deseable es que todos los docentes cuenten con herramientas pedagógicas y digitales para desempeñar sus tareas tanto en la presencialidad, como en la virtualidad. (Ardini, Montserrat Herrera, González Angeletti, \& Secco, 2020)

Reconocer y capturar estas tendencias al diseñar propuestas didácticas, y apoyarnos en ellas para potenciar la enseñanza, sería uno de los desafíos más interesantes del ejercicio de la docencia en nuestra época. Probablemente otro de los desafíos educativos, en cada ciclo y nivel, sería reconstruir lenguajes fragmentados y de construir discursos del mercado que circulan en los medios. Esto incluye reconocer estrategias potenciadoras del aprendizaje colaborativo emergente de la cultura en red, promoviéndolas en la formación docente. Las TIC están sirviendo de base para el surgimiento de un entorno completamente nuevo y diferente dentro del cual tendrán que desenvolverse los procesos de enseñanza y aprendizaje (Brunner, 2003: 43). La escuela debe cambiar porque la sociedad en la que se desenvuelve no es la misma en la que fue creada (Levis, 2008). (De Luca, 2020)

El salto de la educación presencial a los entornos virtuales abrió una puerta a nuevas oportunidades de aprendizaje, pero también a problemas, algunos ya existentes en la presencialidad que se evidenciaron y/o profundizaron en la virtualidad, y otros propios de la nueva modalidad. (Ardini, Montserrat Herrera, González Angeletti, \& Secco, 2020)

Las EVA están propiciando nuevas y distintas formas de aprender mediante el apoyo de los recursos tecnológicos, las posibilidades que ofrecen son múltiples y cada da son más necesarias en un mundo donde prevalece el uso de la tecnología. Esta modalidad es una realidad en diversas instituciones educativas, por tanto, el fomento en el uso de las EVA, ayuda a la materia de historia a ver lo acontecido de una mejor manera, es una necesidad para adecuar las prácticas docentes a las exigencias educativas de hoy, esto supone un cambio en la visión del educador que quiere orientar sus objetivos educacionales al logro del aprendizaje diversificando las estrategias para conseguirlo. (Zambrano Zambrano \& García Vera, 2020)

La desorganización y falta de claridad en linea- 
mientos de trabajo, produce "retrabajo". Las consecuencias las sufrieron docentes y estudiantes. También es importante señalar que las y los docentes se ven fuertemente interpelados por el contexto pandémico y exteriorizan preocupación por la problemática relacionada con su labor y con sus estudiantes. Otro tema relevante que aparece con frecuencia en las respuestas, refiere a cómo construir el vínculo pedagógico, sobre todo con aquellos estudiantes pertenecientes a sectores vulnerables. Algunos docentes manifestaron falta de información sobre la situación particular de sus estudiantes y la sensación de "correr una carrera" contra las desigualdades de origen y el desafío de no profundizarlas en el encuentro digital. La educación va a tener que distinguir -sin oponerlos- entre saberes rentables y saberes indispensables, pues creo que es ahí, en esa encrucijada de saberes, donde se dirime el sentido y el futuro de nuestras escuelas y universidades que se han dado a sí mismas explícitamente un proyecto social, esto es no sólo un oficio, el de enseñar, sino una vocación, la de formar ciudadanos. En este camino se inscribe este aporte, que abre nuevos interrogantes para construir entre todos y todas. (Ardini, Montserrat Herrera, González Angeletti, \& Secco, 2020)

El uso educativo de las Redes Sociales y Entornos Virtuales de Aprendizaje Las aulas virtuales son una nueva modalidad educativa que se desarrolla de manera complementaria o independiente a las formas tradicionales de educación. La posibilidad de gestión de la información tiene un poder relevante en las redes sociales. Esto significa poner al alcance de muchas personas contenidos para fortalecer el conocimiento y el aprendizaje. En este tenor, se resaltó que los microespacios que son creados en la Web 2.0 son de gran valor educativo pues los participantes comparten sus trabajos, sus conocimientos y ayudan a los demás con base en su experiencia, convirtiéndose estos espacios virtuales en centros para la interacción y la comunicación, superando las relaciones cara a cara (Abuin, 2009). (Sornoza Pico \& Yánez Rodriguez, 2020)

Los Entornos Virtuales de Aprendizaje, presentan cuatro características básicas e imprescindibles, que cualquier plataforma de e-learning debería tener, la interactividad, una flexibilidad, escalabilidad o sea la capacidad de la plataforma de e-learning de funcionar igualmente con un número pequeño o grande de usuarios y por último la estandarización (Viñals y Cuenca, 2016). La pandemia obligó a que el sistema educativo de todo el mundo se inclinara por el uso de herramientas virtuales. La interrupción de las instituciones educativas y la ejecución de maneras de educación a distancia han puesto de manifiesto muchas desigualdades estructurales, en las clases sociales, en los niveles regionales, en los tipos de establecimientos. (Sornoza Pico \& Yánez Rodriguez, 2020)

La evidencia respalda que la implementación de un protocolo virtual de clases con la habilitación de plataformas virtuales institucionales como EVA contribuye a solventar y abatir el impacto educativo de la pandemia en la educación. Asimismo, la identificación del poco uso de recursos tecnológicos como Instagram, Microsoft Teams y Blogger, entre otros, parece implicar que, al generarse protocolos virtuales de aprendizaje, estas herramientas son las que presentan menor uso como EVA. Esto indica que aun cuando Microsoft Teams es una herramienta gratuita que puede soportar usos educativos (Microsoft, 2020), el diseño de las plataformas institucionales ha permitido homogeneizar en gran medida la aplicación de este EVA en tiempos de crisis, favoreciendo que los docentes hagan uso de ellas para lograr un mayor seguimiento institucional de la formación educativa de los jóvenes en diferentes niveles. Los docentes no solo se valieron de un EVA en exclusiva para poder continuar con la enseñanza, sino que se apoyaron con diversos recursos para tal objetivo. (Delgado Sánchez \& Martínez Flores, 2021)

Cada vez el uso de los EVA ha ido permeando a las instituciones de educación, y son una excelente herramienta de apoyo en momentos que la educación está en crisis, no solo de pandemia sino también en algunas otras situaciones que pudieran derivarse de otros desastres naturales o sociales. (Delgado Sánchez \& Martínez Flores, 2021)

Las instituciones de educación superior tienen una gran responsabilidad para emular las experiencias profesionales futuras y facilitar la adquisición de un conjunto amplio de competencias. (Hernández-Sellés, 2021)

Las prácticas pedagógicas son procesos vitales para lograr aprendizajes significativos en los estudiantes, y la crisis mundial producida por el COVID-19 ha representado una reorgani- 
zación de éstas en diversos ámbitos, teniendo en cuenta que la valoración de los aprendizajes se realizó en los entornos virtuales. El desarrollo de las clases virtuales se dificultó por diversos motivos entre los que se destacó la conexión a internet, el colapso de la plataforma o la falta de preparación y capacitación en el uso de las TIC. La falta de monitoreo, seguimiento, orientación y entrenamiento para realizar actividades de evaluación podría haber hecho de las evaluaciones un proceso complejo. Las estrategias docentes en entornos virtuales deben fomentar la valoración formativa en la que la participación y el intercambio de ideas sea el eje fundamental para lograr mejores aprendizajes, que, por carencia de instrucciones claras y escasa retroalimentación de los contenidos, produce desmotivación o poco interés en los estudiantes. (Picón, Rodríguez, \& Oliveira)

\section{Conclusiones}

En líneas generales la discusión en el ámbito educativo no se ha centrado en la viabilidad de la educación on- line sino, por el contrario seguir resolviendo los obstáculos a los que se ha enfrentado esta practica educativa, que a 20 años de su publicación por Ortega (2001) siguen siendo las mismas de hoy en día. El autor presenta un análisis sobre las deficiencias y obstáculos más frecuentemente detectados tras analizar las primeras experiencias de enseñanza virtual realizadas en la Universidad de Granada y obtiene

\section{Dificultades derivadas del funcionamiento de los canales de comunicación digital}

- Lentitud en la transmisión de la información, especialmente observable en la recepción de documentos multimedia comprimidos o en tiempo real.

- Interrupción inesperada de la comunicación.

- Coste elevado de las tarifas planas.

- Frecuentes averías en los servidores de información.

- Interrupciones en el suministro eléctrico.

Dificultades derivadas de la calidad tecnológico-educativa de la información

- Obsesión por la generación de contenido literario.
- Descuido en la calidad estética del diseño gráfico y multimedia.

- Excesiva presencia del texto lineal.

- Escasa creatividad y descuido semántico en los textos visuales y muy especialmente en las fotografías.

- Incorrecto planteamiento de los esquemas y gráficos.

- Existencia de ruido comunicativo (deficiente interacción figura-fondo, vocabulario inadecuado, textos visuales borrosos, multimedias desenfocados o con problemas de recepción acústica, etc.).

\section{Dificultades derivadas del diseño metodoló- gico y organizativo de la acción formativa}

- Obsesión por la transmisión de contenido.

- Descuido de objetivos relacionados con la formación social y ética de los ciudadanos.

- Tendencia al uso de metodologías de naturaleza conductista.

- Obsesión por la eficiencia en la adquisición de conocimientos.

- Tendencia a la evaluación de resultados olvidándose en muchos casos el análisis de los procesos de construcción del conocimiento.

- Excesiva tendencia hacia el uso de los sistemas de seguimiento, evaluación y tutorización automática.

- Descuido en el diseño de estrategias instructivas basadas en el diseño de actividades de intercomunicación "muchos a muchos" destinadas al fomento de la creación de conocimiento compartido.

- Desmotivación progresiva y ocasional abandono del proceso de aprendizaje en aquellos casos en los que los diseños metodológicos y organizativos no favorecen el establecimiento de relaciones interpersonales (convivenciales y on line) de alumnos y profesores y de alumnos entre sí." (Belloch, 2012)

Si el ritmo se hubiera mantenido, pues la discusión sería un poco menos avasallante. Pero laa realidad en la que nos ha situado la crisis por la 
pandemia ha obligado que la transición hacia a un nuevo modelo educativo sea abrupta, por lo que esto representa un desafío para todos los componentes de un sistema educativo, docentes, estudiantes, autoridades, y representa un desafío aun mayor superar este panorama en las condiciones de desigualdad social en la que vive Latinoamérica, que hoy más que nunca se ha puesto en evidencia, y cada país de la región deberá buscar el mejor camino para superar las condiciones que se presentan en su realidad, considerando que el retorno a la normalidad como la conocimos, no será posible en su totalidad, como lo piensa cierta parte del colectivo social, paulatinamente debemos asumir que el retorno a las actividades será a una nueva realidad post pandemia, en donde actividades como el teletrabajo en algunos sectores y la educación virtual, lejos de ser desechadas deberán ser fortalecidos ya que serán parte de la nueva normalidad, en la cual es indispensable aprender a enfrentar la incertidumbre, puesto que vivimos en una época cambiante que trae consigo una realidad mucho más compleja, a la cual debemos adaptarnos. (Cóndor Herrera, 2020)

Todo ello no hace más que resaltar los retos a los que aun se enfrenta la comunidad educativa para desarrollar la educación de una manera acorde con las herramientas y oportunidades que brinda el desarrollo constante de la tecnología.

Incluso futuras investigaciones podrían dirigirse a identificar los equipos y dispositivos por medio de las cuales los estudiantes y docentes usan los EVA, ya sean celulares, computadoras portátiles, tabletas y computadoras fijas, y la accesibilidad a internet, para que se puedan ir desarrollando en la educación durante épocas de crisis, así como el conocimiento de espacios que favorezcan su aprendizaje. Esto deja una ventana de oportunidad a las instituciones de educación para realizar una migración de sus contenidos a plataformas virtuales, si bien no se puede virtualizar todos los contenidos y prácticas educativas, sí se pueden generar plataformas de apoyo permanente a la educación, a fin de fortalecer y eventualmente desplazar a la educación tradicional. En futuras investigaciones se tendrán que identificar los EVA que se implementaron en educación básica y los efectos en el aprendizaje de los estudiantes. (Delgado Sánchez \& Martínez Flores, 2021)
El contexto de pandemia ha evidenciado en nuestra sociedad la enorme importancia de la interacción social y también de la organización que ha sido necesaria para articular soluciones. Este gran desafío social debe ayudarnos a revisar y ajustar las propuestas de formación, reconociendo a los estudiantes en una dimensión socioemocional que sustenta la estructuración de los aprendizajes y el desarrollo de competencias curriculares y extracurriculares. (Hernández-Sellés, 2021)

Esto implica engranar la realidad en la que vive cada comunidad para potenciar sus oportunidades y fortalecer las herramientas que le son accesibles para alcanzar los objetivos. La desigualdad es real, la necesidad también, por tanto debe existir un esfuerzo colectivo para mejorar las practicas docentes en el desarrollo de la educación en todos los niveles.

Los entornos virtuales son una herramienta practica y funcional en el soporte de la educación on-line pero su efectividad dependerá del desarrollo de contenido y la generación de las estrategias pedagógicas pertinentes que eviten el desacelere del ritmo con el que se mueve la comunidad educativa en general.

\section{Bibliografía}

Ardini, C., Montserrat Herrera, M., González Angeletti, V., \& Secco, N. E. (julio de 2020). Docencia en tiempos de coronavirus: una mirada al trabajo docente y la experiencia educativa en entornos virtuales en el marco del ASPO por la pandemia COVID-19. Mutual Conexión. Facultad de Ciencias de la Comunicación Universidad Nacional de Córdoba.

Bautista Pérez, G., Borges Sáiz, F., \& Forés i Miravalles, A. (2006). Didactica universitaria en Entornos Virtuales de Enseñanza-Aprendizaje. Madrid: Narcea, S.A de Ediciones.

Belloch, C. (2012). Entornos Virtuales de Aprendizaje. (U. d. Valencia, Ed.) Obtenido de Unidad de Tecnología Educativa .

Cóndor Herrera, O. (2020). Educar en tiempos de Covid-19. CienciAmérica, 9(2).

De Luca, M. P. (2020). LAS AULAS VIRTUALES EN LA FORMACIÓN DOCENTE COMO ESTRATEGIA DE CONTINUIDAD PEDAGÓGICA EN TIEMPOS DE PANDEMIA. USOS Y PARADOJAS. En F. Carolina (Ed.), Análisis Carolina. Serie: Formación Virtual, 33, pág. https://doi.org/10.33960/AC_2020. Madrid.

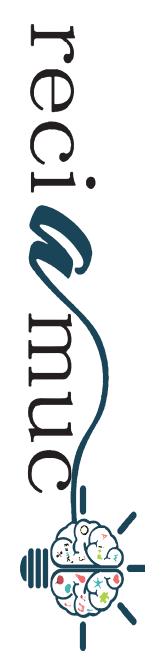


Delgado Sánchez, U., \& Martínez Flores, F. (2021). Entornos virtuales de aprendizaje adoptados en la universidad ante el COVID-19. Diálogos sobre educación , 12(22), DOI: https://doi.org/10.32870/ dse.v0i22.829.

Hernández-Sellés, N. (2021). La importancia de la interacción en el aprendizaje en entornos virtuales en tiempos del COVID-19. Publicaciones, 51(3), 257-275.

Najar S., O., Alvarado G., A., \& Otalora L, J. (2009). ENTORNOS VIRTUALES: UNA MIRADA HACIA LAIMPLANTACIÓN DE GESTIÓN DEL CONOCIMIENTO. AVANCES Investigación en Ingenierí, 11, 73-88.
Picón, G. A., Rodríguez, N., \& Oliveira, A. A. (s.f.). Prácticas de evaluación en entornos virtuales de aprendizajedurante la pandemia COVID-19 y el regreso a la presencialidad. https://preprints.scielo. org/index.php/scielo/preprint/view/2690.

Sornoza Pico, N., \& Yánez Rodriguez, M. (2020). "El uso educativo de redes sociales y entornos virtuales de aprendizaje. Revista Atlante: Cuadernos de Educación y Desarrollo.

Zambrano Zambrano, Y., \& García Vera, C. (junio de 2020). Plan de entornos virtuales de aprendizaje y su aplicación en la asignatura de ciencias sociales en tiempo de pandemia COVID-19 para Estudiantes de bachillerato en Portoviejo, Ecuador. Dominio de las Ciencias, 6(2), 232-245.

\section{CITAR ESTE ARTICULO:}

Yance Tutiven, L. R., Ruiz Salazar, M. E., \& Salazar Palacios, V. del R. (2021).

Entornos Virtuales como soporte en la educación on-line durante la pandemia. RECIAMUC, 5(4), 2-12. https://doi.org/10.26820/reciamuc/5.(4).noviembre.2021.4-12

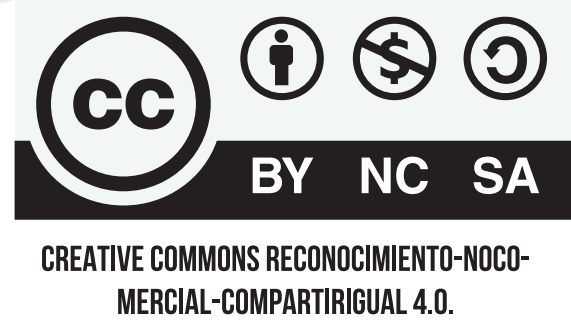

